\title{
Clinical utility gene card for: Hereditary diffuse gastric cancer (HDGC)
}

\author{
Carla Oliveira $^{\star, 1,2}$, Raquel Seruca ${ }^{2,3}$, Nicoline Hoogerbrugge ${ }^{4}$, Marjolijn Ligtenberg ${ }^{4,5}$ and Fátima Carneiro ${ }^{2,3,6}$
}

European Journal of Human Genetics (2013) 21, doi:10.1038/ejhg.2012.247; published online 27 February 2013

\section{DISEASE CHARACTERISTICS}

1.1 Name of the disease (synonyms)

Hereditary diffuse gastric cancer (HDGC); Familial diffuse gastric cancer (FDGC); FDGC and cleft lip with or without cleft palate; and E-cadherin associated hereditary gastric cancer (GC).

\subsection{OMIM\# of the disease}

OMIM\#137215

1.3 Name of the analysed genes or DNA/chromosome segments: Gene: CDH1; cadherin 1, and type 1 E-cadherin (epithelial)

Cytogenetic location: 16q22.1

Genomic coordinates (GRCh37): 16:68 771 194-68 869443 (from NCBI)

\subsection{OMIM\# of the gene(s)}

$\mathrm{OMIM}^{\star} 192090$

\subsection{Mutational spectrum}

Mutation-detection rate: approximately $45 \%$ of all families fulfilling the strict HDGC. ${ }^{1}$

De novo events: these are not precisely known because patients and parents have not yet been systematically tested. CDH1 germline mutations occurred in $7.2 \%$ of apparently sporadic early-onset GC patients invariably with diffuse or mixed histology of GC. From these, proven $C D H 1$-mutation pathogenicity has been assigned only to $2.3 \%$ of the cases that were recurrently diagnosed before 35 years old. ${ }^{2}$

Genomic rearrangements: Deletions of one or multiple $C D H 1$ exons together or not with UTR sequences ( $5^{\prime}$ - and $3^{\prime}$-untranslated regions) occur in $3.8 \%$ of all tested HDGC families. ${ }^{1}$

Nearly 100 different CDH1 germline alterations have been described in more than 120 families. These alterations are distributed throughout the coding, splice-site sequences of the gene, as well as throughout all protein functional domains.

About $15 \%$ of all alterations described have recurrently appeared in one-third of all HDGC families, suggesting derivation of common ancestors and/or mutation hotspots. So far, 12 families have been proven to share a common ancestor (carrying four mutations and one large deletion). ${ }^{1,3}$

Small frameshift insertions and deletions, which occur in $\sim 30 \%$ of all families described so far, are the most frequent mutation type found in $\mathrm{CDH} 1$-associated families, followed by splice-site mutations that occur in $\sim 25 \%$. Nonsense mutations occur in $\sim 20 \%$ and missense mutations occur in another $20 \%$ of the families. Large deletions account for $\sim 4 \%$ of all families, whereas in-frame deletions and germline-promoter methylation are very rare events, accounting for $1 \%$ of $C D H 1$-associated families.

In terms of the predicted impact of all these alterations on the protein structure and function, $80 \%$ of the families encompass alterations that potentially result in protein, due to introduction of premature stop codons, truncation or even complete lack of expression, due to germline $\mathrm{CDH1}$-promoter hypermethylation, as well as complete or partial deletion of the promoter region of the gene together or not with exon 1 and $2^{1}$. The remaining $20 \%$ of the alterations are not expected to lead to protein loss of expression as their impact is expected to change or remove a single amino acid, in the case of missense mutations or in-frame deletions, respectively. For these mutations, functional studies are essential to assess their pathogenicity. ${ }^{4}$

\subsection{Analytical methods}

Stepwise analyses:

Clinical selection: CDH1-mutation analysis should be considered in a family with at least two GC cases, one confirmed diffuse GC (DGC) aged $<50$, or; three confirmed DGC cases in first- or second-degree relatives independent of age, or; personal or family history of DGC and lobular breast cancer (LBC), one diagnosed before the age of 50, or; or an isolated patient with DGC aged $<40$. A careful clinical examination, documented histopathology, and selection of an affected individual in the family, as the proband for genetic screening, are mandatory features for performing costeffective and trustworthy mutation analysis and genetic counseling. ${ }^{5}$

Germline-mutation analysis:

- Sequencing of all coding regions of exons and intron-exon boundaries for point and small indel mutation detection.

- In some centers, pre-screening of the gene by DHPLC and SSCP (genomic level).

- Screening for exon deletions and duplications by multiplex ligationdependent probe amplification (MLPA).

- In case of detection of an unclassified missense variant, assessment of its pathogenicity by:

- Sequencing of at least 50 (100 chromosomes) cancer-free and bona-fide controls.

- In silico assessment of putative effect on splicing, the protein product, and its function.

- Functional analysis by a reference centre (IPATIMUP, Porto Portugal), to test in vitro the impact of the variant in cellinvasion, cell-cell adhesion, and sub-cellular localization.

\footnotetext{
${ }^{1}$ Expression Regulation in Cancer Group, Institute of Molecular Pathology and Immunology of the University of Porto (IPATIMUP), Porto, Portugal; ${ }^{2}$ Faculty of Medicine, University of Porto, Porto, Portugal; ${ }^{3}$ Cancer Genetics Group, Institute of Molecular Pathology and Immunology of the University of Porto (IPATIMUP); ${ }^{4}$ Department of Human Genetics, Radboud University Nijmegen Medical Centre, Nijmegen, The Netherlands; ${ }^{5}$ Department of Pathology, Radboud University Nijmegen Medical Centre, Nijmegen, The Netherlands; ${ }^{6}$ Department of Pathology, Centro Hospitalar de São João, Porto, Portugal

${ }^{*}$ Correspondence: Dr C Oliveira, Expression Regulation in Cancer Group, Institute of Molecular Pathology and Immunology of the University of Porto (IPATIMUP), Rua Dr Roberto Frias s/n, Porto 4200-465, Portugal. Tel: +351 22 5570700; Fax: +351 22 5570799; E-mail: carlaol@ipatimup.pt
} 
- In case of detection of a putative splice-site mutation (missense and intronic):

- RNA analysis to test inactivation of a splice site or activation of cryptic splicing leading to skipping of fragments or complete exons, or retention of intronic portions in the mRNA, frequently generate premature termination of the protein.

- Future perspective: sequencing of the coding regions or the wholegene locus by next-generation sequencing technologies. Use of reliable in vitro tools to test the effect of splice-site mutations, when RNA from $\mathrm{CDH} 1$ mutation carriers is not available.

\subsection{Analytical validation}

Databases such as HGMD (http://www.hgmd.org/); LOVD (http:// www.lovd.nl); and ENSEMBL genome browser (http://www.ensembl.org/) should be used to collect information on CDH1 mutations previously identified, their relationship with a phenotype of HDGC or early-onset diffuse GC, and importantly to assess whether a novel unclassified variant has been detected. In the latter case, only those with a proven pathogenic role should be acknowledged.

Diagnostic testing must be carried out within a certified and accredited laboratory, prepared to decide on the eligibility of the sample to be screened, to evaluate the results obtained, and to write a report with relevant and comprehensive information.

\subsection{Estimated frequency of the disease}

(Incidence at birth ('birth prevalence') or population prevalence) Incidence at birth: nearly $0 \%$.

Population prevalence: $<0.1$ per $100000^{6}$; GLOBOCAN at http:// globocan.iarc.fr/).

Prevalence among GC patients: Less than 1\%. From all GCs that present a family history, $1-3 \%$ will be related to E-cadherin susceptibility, depending on the population analysed (high or low GC incidence geographical areas).

1.9 If applicable, prevalence in the ethnic group of investigated person Not applicable.

\subsection{Diagnostic setting}

\begin{tabular}{lcc}
\hline & Yes & No. \\
A. (Differential) diagnostics & $\bigotimes$ & $\square$ \\
B. Predictive testing & $\square$ & $\square$ \\
C. Risk assessment in relatives & $\Downarrow$ & $\square$ \\
D. Prenatal & $\square$ & $\square$ \\
\hline
\end{tabular}

Comment: A prenatal diagnosis is very rarely requested and, given that the penetrance of the disease in HDGC families carrying deleterious $\mathrm{CDH} 1$ mutations is incomplete, and thus about $20 \%$ of all mutation carriers may never develop clinical disease, this subject should be a theme of extensive discussion during genetic counselling.

\section{TEST CHARACTERISTICS}

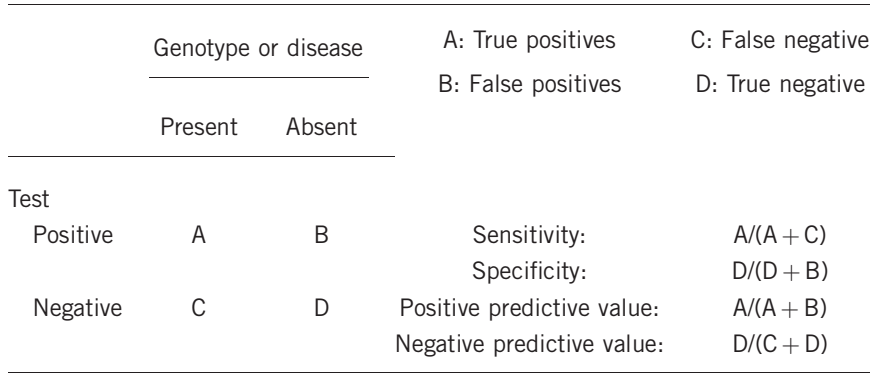

\subsection{Analytical sensitivity}

(proportion of positive tests if the genotype is present)

It has not been determined, nevertheless, we estimate that it will be high (50-75\%), with a combination of direct sequencing and MLPA analyses of the coding regions. Nevertheless, it has been published that $>50 \%$ of $\mathrm{CDH1}$-alteration negative HDGC probands displayed germline $\mathrm{CDH} 1$ allele-specific expression imbalance re-enforcing the existence of unreported defects at the $\mathrm{CDH} 1$ locus. $^{7}$

\subsection{Analytical specificity}

(proportion of negative tests if the genotype is not present)

It has not been determined, nevertheless, we estimate that it will be $>99 \%$, with a combination of direct sequencing and MLPA analyses of the coding regions.

\subsection{Clinical sensitivity}

(proportion of positive tests if the disease is present)

The clinical sensitivity can be dependent on variable factors, such as age or family history. In such cases, a general statement should be given, even if a quantification can only be made case by case.

If the currently accepted clinical criteria are used for patients/ families selection, which considers mainly early-onset of diffuse GC and/or family history of this disease, one can expect that the clinical sensitivity will be lower than the mutation-detection rate among HDGC families, which is $<45 \%$ of all HDGC families when families from different geographic backgrounds are combined. Something that should be pointed out is the fact that if considering a geographical area with low incidence of GC, the mutation-detection rate (clinical sensitivity) will be higher than when considering a geographical area with high or moderate incidence of GC. ${ }^{1,5}$

\subsection{Clinical specificity}

(proportion of negative tests if the disease is not present)

The clinical specificity can be dependent on variable factors, such as age or family history. In such cases, a general statement should be given, even if a quantification can only be made case by case.

It has not been determined, nevertheless, we estimate that it will be $>99 \%$.

\subsection{Positive clinical predictive value}

(life-time risk to develop the disease if the test is positive)

Penetrance in proven mutation carriers is incomplete, and the estimated cumulative risk of diffuse GC and LBC has been calculated. When considering a broad group of $\mathrm{CDH} 1$-mutation carriers, the estimated lifetime risks of diffuse GC was $>80 \%$ in both men and women by age 80 and of LBC was $60 \%$ in women by age $80^{5}$. The combined risk of GC and breast cancer in women has been calculated to be $90 \%$ by age $80 .^{8}$

\subsection{Negative clinical predictive value} (probability not to develop the disease if the test is negative) Assume an increased risk based on family history for a nonaffected person. Allelic and locus heterogeneity may need to be considered.

Index case in that family had been tested:

If a pathogenic germline mutation has been found in the index case, and a first- or second-degree relative does not carry this mutation, the negative clinical predictive value is $>99 \%$. A very rare scenario would be a phenocopy.

Index case in that family had not been tested: 
It is important to highlight that a negative result in a healthy individual from a family with history of HDGC, where a causative mutation has not been identified, does not have any meaning for relatives. Therefore, this approach can best be avoided.

\section{CLINICAL UTILITY}

3.1 (Differential) diagnostics: The tested person is clinically affected

(To be answered if in 1.10 'A' was marked)

\subsubsection{Can a diagnosis be made other than through a genetic test?}

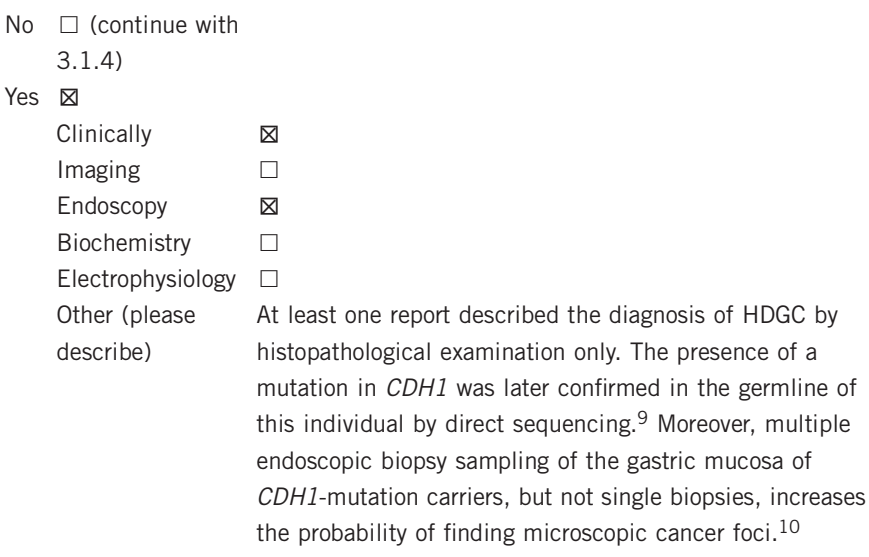

\subsubsection{Describe the burden of alternative diagnostic methods to the patient}

The diagnosis of HDGC can only be made by genetic testing. However, the diagnosis of diffuse GC in a clinically affected person can be established by endoscopic biopsy sampling of the gastric mucosa and subsequent histological scrutiny, which is a burdensome examination. On the other hand, the diagnosis of diffuse GC in a clinically unaffected person can be established by total prophylactic gastrectomy and subsequent histological examination, which is also a burdensome procedure. Nevertheless, almost $100 \%$ of these prophylactic gastrectomies performed in mutation carriers revealed the presence of microscopic cancer foci, and have proven to be curative in asymptomatic $C D H 1$ germline-mutation carriers. ${ }^{5}$

Alternative burdenless diagnostic methods are not available for a clinically affected person.

\subsubsection{How is the cost effectiveness of alternative diagnostic methods to be judged? \\ Not applicable.}

\subsubsection{Will disease management be influenced by the result of a genetic test?}

\section{No. $\square$}

Yes. $\otimes$

Therapy (please describe)
Prophylactic gastrectomy is the only available therapy for HDGC patients and is performed with curative intent. Although the penetrance of HDGC is $80 \%$, microscopic foci of signet ring cells are almost universally present in asymptomatic mutation carriers submitted to prophylactic gastrectomy. If tiny foci of signet ring cell adenocarcinoma or macroscopic gastric carcinoma are detected on gastroscopic biopsies of

\section{Continued}

CDH1-mutation carriers, the IGCLC recommends patients to undergo total gastrectomy. ${ }^{5}$ The Dutch working group on hereditary GC further recommends extensive lymphadenectomy in case of preoperatively diagnosed invasive $\mathrm{GC}^{11}$

The surgical procedure consists of total gastrectomy with Roux-en-Y esophagojejunostomy. The distal margin should be at least $1.0 \mathrm{~cm}$ below the pyloric region to ensure resection through duodenal mucosa. Before performing the reconstruction, a frozen section procedure of the proximal margin is warranted to confirm that no gastric cardia mucosa is left behind and allowing direct re-resection. This measure is recommended because any residual gastric mucosa may increase the risk of subsequent (pre)malignant lesions, and inspection of the z-line may not in all cases be sufficient to achieve proximal clearance. ${ }^{5,11}$ Other non-surgical therapy regimens used in sporadic GC cases, with similar clinical presentation, may also apply for GC patients carrying a $C D H 1$ mutation.

Women carrying a $\mathrm{CDH} 1$ mutation are at higher risk to develop LBC with lifetime risk of $60 \%$ by the age of 80 years, rising from 40 years of age. ${ }^{5}$ Annual mammography and breast MRI is recommended from the age of 35 onwards. ${ }^{5}$ For some women, prophylactic mastectomy may be a reasonable option, although current data are insufficient to infer efficacy of both surveillance and prophylactic breast surgery in patients carrying a $\mathrm{CDH} 1$ mutation. 5,12,13

Prognosis (please describe)

The prognosis of asymptomatic patients that underwent a prophylactic gastrectomy is very good. In contrast, patients who develop symptomatic invasive diffuse gastric carcinoma, even before the age of 40 , have a poor prognosis with as few as $10 \%$ having early and curable disease. A deeper knowledge of the natural history of intramucosal cancer foci, in asymptomatic carriers of $\mathrm{CDH} 1$ mutations, is necessary to evaluate the possibility of safely postponing gastrectomy, while submitting patients to a program of intensive gastroscopy. In light of the current knowledge, it is safer to recommend prophylactic gastrectomy early in the adult life of asymptomatic mutation carriers.

There are implications for decision on and long-term follow-up of prophylactic gastrectomy patients, namely iron deficiency anaemia (mainly in women), psychological and physical fitness, occupation and other family commitments, as well as physiological, metabolic and emotional impact of removing a young adults' stomach, which re-enforced the need for multidisciplinary teams when managing these patients. $\mathrm{CDH} 1$-mutation female carriers of childbearing age, who choose to undergo prophylactic gastrectomy, should be counselled that pregnancies occurring post-gastrectomy will have specific nutritional requirements. ${ }^{14}$

CDH1-mutation carriers who do not (yet) want to undergo a prophylactic gastrectomy, as well as individuals with $50 \%$ risk of being carriers, who are not (yet) willing to be tested for the mutation, are advised an intensive surveillance program with endoscopy and multiple biopsies, according to IGCLG guidelines. ${ }^{5}$ Diffuse GC is difficult to detect at an early and treatable stage because of difficulties to identify (sub)mucosal 
lesions and biases in sampling in macroscopically normal-appearing gastric mucosa. ${ }^{15}$ Such biopsies need to be evaluated by pathologists with expertise in this field. Several studies have shown that even though CDH1-mutation carriers had negative biopsies before prophylactic gastrectomy, cancer foci were detected in their gastrectomy specimens. ${ }^{16,17}$

Management (please describe) Genetic counselling is an essential component of the evaluation and management of HDGC.

The genetic evaluation should include a careful threegeneration family pedigree, histopathological confirmation of diffuse GC diagnoses or precursor lesions, a discussion of lifetime risks of diffuse GC, and current CDH1-mutation-detection rates. Informed consent for genetic testing is required. The counselling process should include also input from a multidisciplinary team comprising those with relevant expertise in gastric surgery, gastroenterology, pathology, and nutrition, ideally in both the pre-genetic and post-test settings. Genetic testing should be initiated in an affected proband. ${ }^{5}$ Consideration of genetic testing usually starts from age $18,{ }^{18}$ although the overall risk of diffuse GC before the age of 20 is very low. Genetic testing below age 18 is usually not advised because GC almost always develops at an adult age.

3.2 Predictive Setting: The tested person is clinically unaffected but carries an increased risk based on family history

(To be answered if in 1.10 ' $\mathrm{B}$ ' was marked)

\subsubsection{Will the result of a genetic test influence lifestyle and prevention?}

If the test result is positive (please describe):

CDH1-mutation-positive patients with normal gastric biopsies should be advised to undergo gastrectomy once the genetic testing results are known and once individuals are older than 20 years. The timing of this operation may also vary according to the preferences, as well as the physical and psychological fitness of the individual. In patients going forward for gastrectomy, a baseline endoscopy should be performed before surgery to look for macroscopic tumour and in order to inform the data on endoscopic detection of microscopic lesions. For individuals in whom gastrectomy is not currently being pursued (for example, through patient choice), annual gastroscopy with multiple blind biopsies should be offered in order to ensure that there is no evidence of clinically significant lesions and for research purposes. $^{5}$

For females, annual breast cancer surveillance is recommended starting at age 35 to detect breast cancer at an early stage. Some women might choose for prophylactic mastectomy. 5,11

In some cases, a positive result may influence family planning, namely considering offspring.

If the test result is negative (please describe)

In a family with a detected $C D H 1$ mutation, discharge from intensive screening program that will contribute to psychological relief.

3.2.2 Which options in view of lifestyle and prevention does a person at risk have if no genetic test has been done (please describe)?

In case of $50 \%$ risk to carry a $C D H 1$ mutation that runs in the family, the same options as those presented for $\mathrm{CDH1}$-mutation carriers that chose not to have a prophylactic gastrectomy. No data are available on the outcome of prophylactic gastrectomies done in asymptomatic people at risk not screened for $C D H 1$ mutations. It is nevertheless expected that given the current mutation-detection rate for HDGC patients, as well as the autosomal pattern of inheritance of the disease (50\% chance of not inheriting the mutant allele), such options would be ineffectual in about half of these persons at risk.

People at risk that chose not to be tested for $\mathrm{CDH} 1$ mutations, but have a family history of HDGC, may discuss with clinicians and genetic counsellors the implementation of lifestyle and preventive measures described above for CDH1-mutation carriers. The cost/ benefit for such patients should be strongly debated, mainly in what concerns invasive procedures, such as prophylactic surgery.

3.3 Genetic risk assessment in family members of a diseased person (To be answered if in 1.10 ' $\mathrm{C}$ ' was marked)

\subsubsection{Does the result of a genetic test resolve the genetic situation in that family?}

Yes, it will define an autosomal-dominant inheritance if the mutation is known in the family.

\subsubsection{Can a genetic test in the index patient save genetic or other} tests in family members?

Yes, if the result is negative or uncertain, testing of family members should not be recommended. Recommendation for screening applies only to mutation carriers and persons at risk.

\subsubsection{Does a positive genetic test result in the index patient enable a predictive test in a family member? \\ Yes.}

\subsection{Prenatal diagnosis}

(To be answered if in 1.10 'D' was marked)

\subsubsection{Does a positive genetic test result in the index patient enable a prenatal diagnosis?}

Technically feasible, generally either not recommended or not well accepted by parents, because of an incomplete penetrance and adult onset of the disease. This may vary among different countries, namely specific laws and ethical values.

\section{IF APPLICABLE, FURTHER CONSEQUENCES OF TESTING}

Please assume that the result of a genetic test has no immediate medical consequences. Is there any evidence that a genetic test is nevertheless useful for the patient or his/her relatives? (Please describe)

Support for family life organisation.

Efficiency of subsequent clinical management.

For many patients, prove of diagnosis is a value itself - irrespective of a medical benefit - because the disease and its cause can clearly be identified.

Although there is no cure for HDGC syndrome, the diagnosis helps to guide appropriate medical management. For asymptomatic carriers, this information is particularly important because it will implicate guidance through options of prophylactic gastrectomy or frequent endoscopy surveillance. Treatment for patients with clinical presentation of the disease is similar to that of sporadic GC patients.

An affected person/asymptomatic mutation carrier can learn that his or her children may develop the disease, but also that they have $50 \%$ probability of being noncarriers. 


\section{CONFLICT OF INTEREST}

The authors declare no conflict of interest.

\section{ACKNOWLEDGEMENTS}

This work was supported by EuroGentest2 (Unit 2: 'Genetic testing as part of health care'), a Coordination Action under FP7 (Grant Agreement Number 261469) and the European Society of Human Genetics. This study was also supported by the Portuguese Foundation for Science and Technology (FCT) (Projects: PTDC/SAU-GMG/110785/2009 and PTDC/SAU-ONC/110294/ 2009); and by Calouste Gulbenkian Foundation.

1 Oliveira C, Senz J, Kaurah $\mathrm{P}$ et al: Germline $\mathrm{CDH} 1$ deletions in hereditary diffuse gastric cancer families. Hum Mol Genet 2009; 18: 1545-1555.

2 Corso G, Pedrazzani C, Pinheiro $\mathrm{H}$ et al: E-cadherin genetic screening and clinicopathologic characteristics of early onset gastric cancer. Eur J Cancer 2011; 47: 631-639.

3 Kaurah P, MacMillan A, Boyd $\mathrm{N}$ et al: Founder and recurrent $C D H 1$ mutations in families with hereditary diffuse gastric cancer. JAMA 2007; 297: 2360-2372.

4 Suriano G, Oliveira C, Ferreira $\mathrm{P}$ et al: Identification of $\mathrm{CDH} 1$ germline missense mutations associated with functional inactivation of the E-cadherin protein in young gastric cancer probands. Hum Mol Genet 2003; 12: 575-582.

5 Fitzgerald RC, Hardwick R, Huntsman D et al: International Gastric Cancer Linkage Consortium: hereditary diffuse gastric cancer: updated consensus guidelines for clinical management and directions for future research. J Med Genet 2010; 47: $436-444$.

6 Oliveira C, Seruca R, Carneiro F: Hereditary gastric cancer. Best Pract Res Clin Gastroenterol 2009; 23: 147-157.
7 Pinheiro H, Bordeira-Carriço R, Seixas S et al: Allele-specific CDH1 downregulation and hereditary diffuse gastric cancer. Hum Mol Genet 2010; 19: 943-952.

8 Pharoah PD, Guilford P, Caldas C: International Gastric Cancer Linkage Consortium: incidence of gastric cancer and breast cancer in $\mathrm{CDH1}$ (E-cadherin) mutation carriers from hereditary diffuse gastric cancer families. Gastroenterology 2001; 121: 1348-1353.

9 Oliveira C, Moreira H, Seruca R, de Oliveira MC, Carneiro F: Role of pathology in the identification of hereditary diffuse gastric cancer: report of a Portuguese family. Virchows Arch 2005; 446: 181-184.

10 Barber ME, Save V, Carneiro F et al: Histopathological and molecular analysis of gastrectomy specimens from hereditary diffuse gastric cancer patients has implications for endoscopic surveillance of individuals at risk. J Pathol 2008; 216: 286-294.

11 Kluijt I, Sijmons RH, Hoogerbrugge $\mathrm{N}$ et al: Familial gastric cancer: guidelines for diagnosis, treatment and periodic surveillance. Fam Cancer 2012; 11: 363-369.

12 Kurian AW, Sigal BM, Plevritis SK: Survival analysis of cancer risk reduction strategies for BRCA1/2 mutation carriers. J Clin Oncol 2010; 28: 222-231.

13 Hoogerbrugge $\mathrm{N}$, Kamm YJ, Bult $\mathrm{P}$ et al: The impact of a false-positive MRI on the choice for mastectomy in BRCA mutation carriers is limited. Ann Oncol 2008; 19: 655-659.

14 Kaurah P, Fitzgerald R, Dwerryhouse S, Huntsman DG: Pregnancy after prophylactic total gastrectomy. Fam Cancer 2010; 9: 331-334.

15 Fitzgerald RC, Caldas C: Clinical implications of E-cadherin associated hereditary diffuse gastric cancer. Gut 2004; 53: 775-778.

16 Huntsman DG, Carneiro F, Lewis FR et al: Early gastric cancer in young, asymptomatic carriers of germ-line E-cadherin mutations. N Engl J Med 2001; 344: 1904-1909.

17 Carneiro F, Huntsman DG, Smyrk TC et al: Model of the early development of diffuse gastric cancer in $\mathrm{E}$-cadherin mutation carriers and its implications for patient screening. J Pathol 2004; 203: 681-687.

18 Kluijt I, Siemerink EJ, Ausems MG et al: on behalf of the Dutch Working Group on Hereditary Gastric Cancer. $\mathrm{CDH} 1$-related hereditary diffuse gastric cancer syndrome: Clinical variations and implications for counseling. Int J Cancer 2011; 131: 367-376. 